\title{
Spontaneous pattern formation in driven nonlinear lattices
}

\author{
Andrea Vanossi ${ }^{1,2,4}$, K. Ø. Rasmussen ${ }^{1}$, A. R. Bishop ${ }^{1}$, Boris A. Malomed ${ }^{3}$, and V. Bortolani ${ }^{4}$ \\ ${ }^{1}$ Center for Nonlinear Studies and Theoretical Division, Los Alamos National Laboratory, Los Alamos, New Mexico 87545 \\ ${ }^{2}$ Dipartimento di Fisica, Università di Bologna, V.le Berti Pichat 6/2, I-40127, Bologna, Italy \\ ${ }^{3}$ Department of Interdisciplinary Studies, Faculty of Engineering, Tel Aviv 69975, Israel \\ ${ }^{4}$ INFM e Dipartimento di Fisica, Università di Modena, Via Campi 213/A, 41100 Modena, Italy
}

(October 29, 2018)

\begin{abstract}
We demonstrate the spontaneous formation of spatial patterns in a damped, ac-driven cubic KleinGordon lattice. These patterns are composed of arrays of intrinsic localized modes characteristic for nonlinear lattices. We analyze the modulation instability leading to this spontaneous pattern formation. Our calculation of the modulational instability is applicable in one and two-dimensional lattices, however in the analyses of the emerging patterns we concentrate particularly on the twodimensional case.
\end{abstract}

Complex spatial patterns are often observed in systems driven away from equilibrium [1]. Typically, the patterns emerge when relatively simple systems are driven into unstable states that will deform dramatically in response to small perturbations. As the patterns are arising from an instability, the pattern-forming behavior is likely to be extremely sensitive to small changes in system parameters. The description of deterministic pattern forming systems is typically accomplished in the form of partial differential equations such as the Navier-Stokes equations for fluids and reaction-diffusion equations for chemical systems. These phenomena have primarily been studied exclusively in continuum systems such as hydrodynamical, optical, chemical systems and liquid crystals, although more recently pattern formation of similar type have also been reported in periodically vibrated granular media [2].

Complementing the development of the theoretical understanding of pattern formation in continuum systems, the localized mode forming ability of discrete lattices has also received significant recent attention. There is now a fairly complete understanding of the existence and stability of localized structures (often referred to as intrinsic localized modes (ILM's) or discrete breathers) in a variety of nonlinear lattices, undriven [3] as well as driven [4]. It is fair to claim that these collective patterns are well understood while the process of their creation and interaction remains relatively unexplored.

In the present communication we study the pattern forming abilities of a damped and periodically driven nonlinear lattice. Specifically, we demonstrate how a driven nonlinear (cubic) Klein-Gordon lattice forms a variety of patterns via modulational instabilities. We analyse the modulational instabilities and show how these relate to the length-scale of the patterns that are formed. Normally, the spatial extend (characteristic length-scale) of ILM's is directly related to the frequency of the ILM's [3]. However, in our case the (generally different) lengthscale emerging from the instability may lead to a lengthscale competion, the results of which we will explore.
Model and stability of homogeneous solution. First, we study the Klein-Gordon lattice

$$
\ddot{x}_{n}+\gamma \dot{x}_{n}+\omega_{0}^{2} x_{n}=\Delta_{n} x_{n}+\lambda x_{n}^{3}+\epsilon \cos \omega t,
$$

where $\gamma$ is the damping parameter, $\omega_{0}$ the natural frequency of the oscillators, $\lambda= \pm 1$ the nonlinearity parameter and finally $\epsilon$ is the amplitude of the ac-drive at frequency $\omega$. In one dimension the nearest neighbor coupling is $\Delta_{n} x_{n}=x_{n+1}-2 x_{n}+x_{n-1}$ [5]. The amplitude $A_{0}$ (and phase $\delta_{0}$ ) of the spatially homogeneous solution $x_{n}=A_{0} \cos \left(\omega t+\delta_{0}\right)$ of Eq.(1) can, within the rotating wave approximation, be shown to satisfy

$$
A_{0}^{2}\left(\gamma^{2} \omega^{2}+\left(\omega^{2}-\omega_{0}^{2}+\frac{3}{4} \lambda A_{0}^{2}\right)^{2}\right)=\epsilon^{2} .
$$

The amplitude $A_{0}$ of the response to a driving amplitude $\epsilon$ is shown for the soft $(\lambda=1)$ potential in Fig. 1.

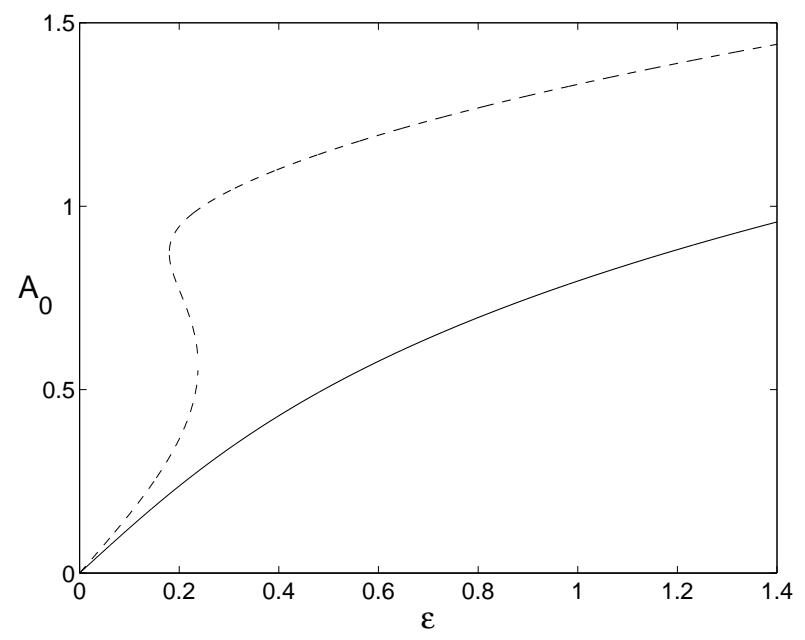

FIG. 1. Amplitude $A_{0}$ of response vs driving amplitude $\epsilon$ of the ac-drive at frequency, $\omega=1.2 \omega_{0}$ (solid line) and $\omega=0.8 \omega_{0}$ (dashed line). Remaining parameters are $\omega_{0}=1.3$, $\gamma=0.15 \omega_{0}$, and $\lambda=1$ (soft potential). 
For $\omega<\omega_{0}$ (dashed line) three solutions are possible, while for $\omega>\omega_{0}$ (solid line) a single solution is possible. A similar picture is valid for the $\operatorname{hard}(\lambda=-1)$ potential except that the multiple solutions then appear in the case $\omega>\omega_{0}$.

Analyzing the stability of the homogeneous solution with respect to spatial perturbations, we introduce $x_{n}=$ $y+z_{n}$ into Eq.(11). Assuming periodic boundary conditions, we may expand $z_{n}$ in its Fourier components $z_{n}=\sum_{k} \exp (i k n) \xi_{k}(t)$, where the mode amplitude $\xi_{k}(t)$ is then governed by

$$
\ddot{\xi}_{k}+\gamma \dot{\xi}_{k}+\omega_{k}^{2} \xi_{k}=\frac{3}{2} \lambda A_{0}^{2}\left[1+\cos \left(2 \omega t+2 \delta_{0}\right)\right] \xi_{k}
$$

with $\omega_{k}^{2}=\omega_{0}^{2}+4 \sin ^{2}(k / 2)$ denoting the linear dispersion relation of the system.

Finally, the transformation $\xi_{k}(t)=\zeta_{k}(\omega t+$ $\left.\delta_{0}\right) \exp \left(-\frac{\gamma}{2}(\omega t+\delta)\right) \equiv \zeta_{k}(\tau) \exp \left(-\frac{\gamma}{2} \tau\right)$ reduces Eq. (3) to a standard Mathieu equation

$$
\frac{d^{2} \zeta_{k}}{d \tau^{2}}+a \zeta_{k}-2 q \cos (2 \tau) \zeta_{k}=0
$$

where

$$
a=\frac{1}{4 \omega^{2}}\left(4 \omega_{k}^{2}-6 \lambda A_{0}^{2}-\gamma^{2}\right) \quad \text { and } \quad q=\frac{3 \lambda A_{0}^{2}}{4 \omega^{2}} .
$$

As is well-known [6] the Mathieu equations exhibit parametric resonances when $\sqrt{a} \simeq i$, where $i=1,2,3, \ldots$. The width of the resonance regions depends on the ratio $q / a$ (see, e.g. [7]). In the framework of Eq.(仹 the extent of the primary resonance $a \simeq 1$ can easily be estimated to be $(a-1)^{2}<q^{2}$. However, in the presence of the damping $\gamma$ the resonance condition for Eq.(3) becomes

$$
q^{2}>\frac{\gamma^{2}}{\omega^{2}}+(a-1)^{2}
$$

With $a$ and $q$ defined in Eqs.(5), given $\lambda, \gamma, \omega, \omega_{0}$, and $\epsilon$, this translates into an instability band of certain wavenumbers $k$.

Figure 2 shows this instability band as given by Eq.(6) for parameters corresponding to the solid curve in Fig. 1. The shaded region is the band of wavenumbers that are unstable according to Eq.(6) and the dashed line indicates the most unstable wavenumber, i.e. $a=1$. The effect of the damping clearly is to pinch off the instability region at a finite driving $\epsilon>0$. Similar, using Eq.(6), instability regions can be determined for the solutions indicated by the dashed curve in Fig.1.

We have verified the presence and location of the instability band by direct simulations of Eq.(1) starting from the initial condition $x_{n}(t=0)=A_{0} \cos \left(\delta_{0}\right)+\eta_{n}$, where, $\eta_{n}$, represents a small $\left(\left|\eta_{n}\right| \ll A_{0}\right)$ spatially random perturbation. This initial condition injects energy into all wavenumbers and in the presence of an unstable region of wavenumbers the dynamics will enhance the energy content in this region and thereby identify the unstable region.

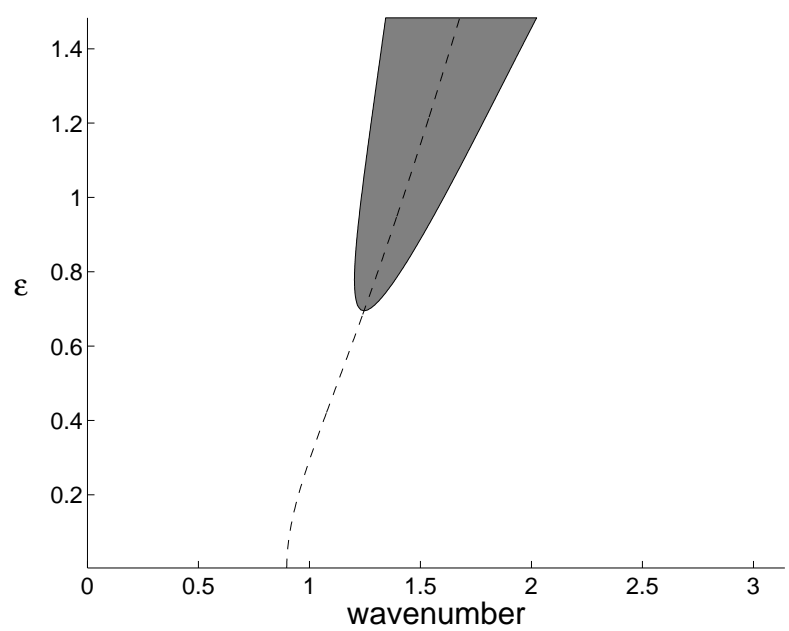

FIG. 2. Instability region (shaded) in the $(\epsilon, k)$-plane. Dashed line indicates the most unstable wavenumber $a=1$. Parameters corresponds to the solid line in Fig. 1.

The above analysis is easily extended into the case of two spatial dimensions [5], the only required change being that the dispersion relation now is $\omega_{\vec{k}}^{2}=\omega_{0}^{2}+$ $4 \sin ^{2}\left(k_{x} / 2\right)+4 \sin ^{2}\left(k_{y} / 2\right)$, where the wavevector is $\vec{k}=$ $\left(k_{x}, k_{y}\right)$. The instability in this case appears on an annulus in the wavevector plane, with a radius given by $a=1$ (see, Eq.(5)) and a width determined by Eq.(6).

Pattern formation. Numerical simulations allow us not only to verify the predicted instability band, but also to follow the full nonlinear development and saturation initiated by the instability. In particular, in regions of parameter space we obtain the spontaneous formation of patterns of distinct spatial geometry. Although we have observed this phenomenon in one as well as in two dimensions, in the present communication we focus on the two-dimensional system, where the pattern formation is very rich.

Although the dynamics show different features according to the specific region of parameter space, it is possible to trace a typical behavior as follows: Initializing the system in the spatially homogeneous state described above with a small amount of randomness added, the instability sets in after a certain number of cycles of the ac-drive, depending on the strength of the parametric resonance, i.e. on the value of $\sqrt{a} \simeq 1,2,3, \ldots$. Thereafter the system usually evolves through a sequence of different patterns (rhombi, stripes, etc.), composed of localized regions of high amplitude oscillations, before reaching a final configuration that may or may not result in a structure of definite symmetry.

Due to the sensitive response to very small changes of the parameters, determining stability regions for the different pattern geometries is a difficult task. However, in 
the case of a hard potential $(\lambda=-1)$, Fig. 3 shows a limited area of $(\epsilon, \omega)$-space in which distinct spatial patterns emerge and remain stable.

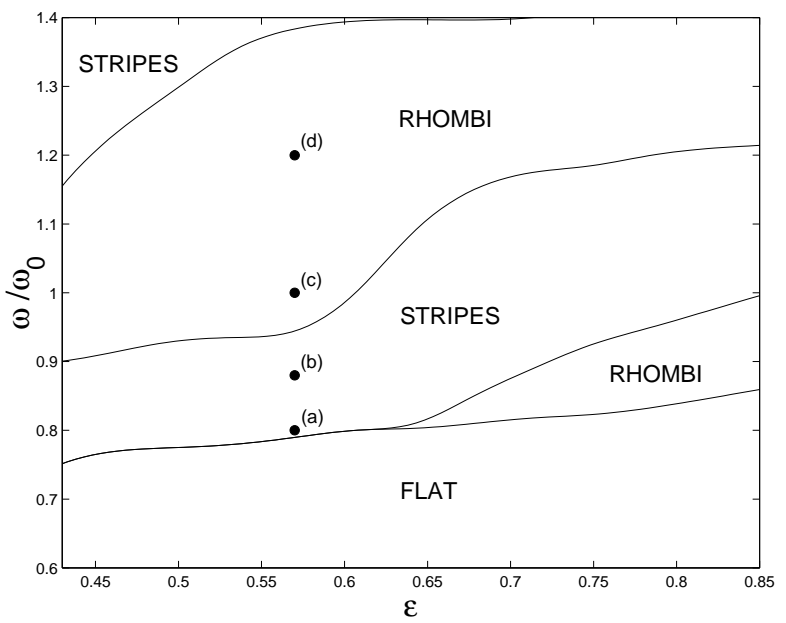

FIG. 3. Stability diagram showing the stability boundaries between the possible patterns in the $(\epsilon, \omega)$-plane. Parameters are $\omega_{0}=0.75, \lambda=-1$ (hard potential), $\gamma=0.05 \omega_{0}$. The four points refer to the specific spatial structures shown in Fig. 4.

This diagram is constructed following the full dynamics of the system for thousands of cycles. As our study concentrates on patterns arising from instabilities of the homogeneous solutions, we do not discuss possible hysteretic behavior as is sometimes observed in similar systems (see, e.g. Ref.[2]).

Figure 4 shows representative examples of the spontaneously emerging patterns corresponding to the points marked in Fig. 3 .

FIG. 4. (Color) Spatial patterns corresponding to the four points marked in Fig. 3 for $\epsilon=0.57$ : (a) straight stripes $\left(\omega=0.8 \omega_{0}\right)$, (b) modulated stripes $\left(\omega=0.88 \omega_{0}\right)$, (c) rhombi $\left(\omega=1.0 \omega_{0}\right)$, (d) localized rhombi $\left(\omega=1.2 \omega_{0}\right) . x_{n, m}$ is plotted along the vertical axes.
The patterns consist of localized regions of high amplitude oscillations (ILM's) residing on a background that oscillates at the frequency, $\omega$, of the ac-drive. In all the considered cases we have observed the natural result that patterns are only energetically sustained when the ILM, $\omega_{I L M}$, and driving, $\omega$, frequencies are commensurate, i.e. $\omega_{I L M}=n \omega$, where $n$ is an integer. For the patterns displayed in Fig. $4, n=2$. Further, the motion of the ILM's is out of phase, i.e. , at the points in time where the background oscillation reaches its maximal excursion the ILM's obtain their minimal amplitude such that at these points the state is completely homogeneous.

At a fixed driving $\epsilon$, for increasing values of the frequency $\omega$, as in Fig. 4, we can observe the following behavior: Due to the presence of the damping $\gamma$, at sufficiently small $\omega$ the spatially homogeneous solution is stable towards all possible spatial modulations such that the flat state is sustained. However, for values near point (a) the system becomes unstable with respect to certain spatial modulations and spatial patterns in the form of large stripes emerge (Fig. 4(a)). Increasing the frequency, these stripes become thinner and denser and begin to show an increasingly clearer modulation (Fig. $4(\mathrm{~b}))$. The characteristic length scale of these patterns is set by the size of the unstable $\vec{k}$-vector according to the above analysis. The nonlinear character of the system results in a transition towards a more isotropic geometry (rhombic) as the driving frequency is increased further (Fig. 4(c)). As in the case of the stripes, stronger localization of the ILM's arranged in the rhombic pattern (Fig. 4(d)) is observed for even larger driving frequencies. The angle between the sides of the rhombus unit cell varies but for the hard potential it is always close to $\pi / 2$. For values of $\epsilon$ larger than those displayed in Fig. 3, the final mesoscopic patterns of the system dynamics are spatially disordered much like the phenomena observed in granular media [2]. It is important to realize that the length of the unstable wavevector determines the lengthscales of the final patterns, while the symmetry of the patterns is determined by the nonlinear character of the system.

As a result of the periodic boundary conditions, the length scale of the emerging patterns must be commensurate with the system size. However, we have observed that the existence of a band (variability in length and angle) of unstable $\vec{k}$-vectors (see Fig. 2 and related discussion) allows continuous accommodation of this constraint except for the discontinuous changes in length scales occurring when it is energetically favorable for the system to add (or subtract) an additional stripe (or row of ILM's).

For the soft potential $(\lambda=1)$ the variation of the amplitude $\epsilon$, and the frequency, $\omega$ of the ac-drive is particularly problematic as the dynamics in this case can lead to the development of catastrophic instabilities as 
one or several oscillators overcome the finite barrier in the quartic potential.In all the cases we have been able to simulate, the early stage time evolution of the system is characterized by the formation of ILM's regularly arranged in a square pattern. This spatial configuration, which is sustained for up to hundreds of cycles, seems always to suffer from a weak instability and eventually deforms into a rhombic pattern, as shown in Fig. 5.

FIG. 5. (Color) Snapshot of spontaneously formed pattern of ILM's. The red line represents a one-dimensional cut of the pattern array, whose dynamics we have analyzed in detail (see Fig. 6).

Contrasting with the case of the hard potential, here in the soft potential the angle between the sides of the rhombus unit cell is always close to $2 \pi / 3$ (so almost hexagonal).

We now analyze this pattern more closely. The pattern shown in Fig. 5 consists of ILM's spontaneously organized into a regular rhombic pattern. The ILM's are spatially localized and perform harmonic temporal oscillations (at the frequency, $\omega$, of the ac-drive) and are therefore objects that are well described 30 in the literature. A particular feature of these ILM's is that they reside on a background that oscillates at the same frequency. To expose the dynamics of the ILM's more closely, we show in Fig. 6 a time sequence along a onedimensional cut of the two-dimensional system (the cut is indicated by the line in Fig. 5). It should be noted that in Fig. 6 we have removed the oscillations of the background in order to expose the ILM dynamics most clearly.

Although we have focused on the ILM dynamics in the case of a soft potential, the features are analogous in the case of the hard potential and only the symmetry of the patterns is different.
FIG. 6. ILM dynamics monitored along the direction indicated by the line in Fig. 5 .

In summary we have studied the modulational instability in the damped and ac-driven cubic nonlinear KleinGordon lattice. The analysis applies to one as well as two spatial dimensions. We have further demonstrated how these instabilities lead to a variety of mesoscopic patterns of intrinsic local modes. In the case of a hard potential we characterized the patterns in a stability diagram and in the case of the soft potential we showed that the dynamics always result in a rhombic pattern with an angle close to $2 \pi / 3$. These rhombic patterns were never observed in the case of the hard potential. This difference in the shapes of the rhombic patterns in the hard and soft cases can be understood by exploiting the analogy between the changes in the steady states of a dissipative systems and phase transitions in systems at thermodynamic equilibrium (see. e.g. Ref. [8]). In terms of this analogy the appearance of spatially periodic structures in a nonequilibrium system can be connected to a perturbation of the translational symmetry of thermodynamic states in equilibrium. A study based on this philosophy was pursued in Ref. [8] with the results that the angle defining rhombic patterns is given by the coefficient of the cubic term in the system, which is precisely our observation. From the present analysis it appears that experimental studies of the pattern forming abilities of discrete systems present an excellent opportunity to study ILM's and their mutual interactions and mesoscopic patterning. For example, optical systems [9] and spin systems [10] appear good candidates for such studies.

Research at Los Alamos National Laboratory is performed under the auspice of the US DoE. 
[1] M. C. Cross and P. C. Hohenberg, Rev. Mod. Phys. 65, 851 (1993).

[2] F. Melo, P. Umbanhowar, and H. L. Swinney, Phys. Rev. Lett. 72, 172 (1994); 75, 3838 (1995);P. Umbanhowar, F. Melo and H. L. Swinney, Nature, 382, 793 (1996); C. Bizon et al., Phys. Rev. Lett. 80, 57 (1998).

[3] S. Aubry, Physica D 103, 201 (1996); S. Flach and C. R. Willis, Phys. Rep. 295, 182 (1998); D. Hennig, and G. P. Tsironis, Phys. Rep. 307, 335 (1999).

[4] J. L. Marin and S. Aubry, Nonlinearity 9, 1501 (1994); B. A. Malomed, Phys. Rev. B 49, 5962 (1994).

[5] The two-dimensional version of Eq.(11) is $\ddot{x}_{n, m}+\gamma \dot{x}_{n, m}+$ $\omega_{0}^{2} x_{n, m}=x_{n+1, m}-4 x_{n, m}+x_{n-1, m}+x_{n, m-1}+x_{n, m+1}+$ $\lambda x_{n, m}^{3}+\epsilon \cos \omega t$, where the parameters have the interpretation given in connection with Eq. (便).

[6] V. I. Arnold, Mathematical Methods of Classical Mechanics, (Springer, New York, 1997).

[7] M. Abramowitz and I. A. Stegun, Handbook of mathematical functions with formulas, graphs, and mathematical tables, (U.S. Govt. Print. Off., Washington, D.C., 1964).

[8] B. A. Malomed, A. A. Nepomnyashcii, and M. I. Tribekskii, Zh. Eksp. Teor. Fiz 96, 684 (1989) [Sov. Phys. JETP 69388 (1989)].

[9] H. S. Eisenberg, Y. Silberberg, R. Morandotti, A. R. Boyd, J. S. Aitchison, Phys. Rev. Lett. 81, 3383 (1998).

[10] R. Lai, and A. J. Sievers, Phys. Rev. Lett. 81, 1937 (1998). 
This figure "fig4.gif" is available in "gif" format from: http://arxiv.org/ps/nlin/0004035v1 
This figure "fig5.gif" is available in "gif" format from: http://arxiv.org/ps/nlin/0004035v1 
This figure "fig6.gif" is available in "gif" format from: http://arxiv.org/ps/nlin/0004035v1 